\title{
Prioritizing Alarms in Real-Time: Intelligent Alarms
}

\section{Advanced System}

\author{
Eduardo Navarra Satuf ${ }^{1}$, Mario Cesar Mello Massa de Campos $^{1}$ and Roberto Schirru ${ }^{2}$ \\ 1. Petrobras Research and Development Centre, Rio de Janeiro 21941-915, Brazil \\ 2. Alberto Luiz Coimbra Institute for Graduate Studies and Researchin Engineering, Federal University of Rio de Janeiro, Rio de \\ Janeiro 21941-972, Brazil
}

Received: January 08, 2015 / Accepted: January 21, 2015 / Published: February 25, 2015.

\begin{abstract}
During the operation of complex process, such as oil production or refining, abnormal situations may occur, leading to an alarm flooding. Alarm flooding is the signalling of a large number of alarms in a few minutes, in such a way that it is impossible for the operator to attend to all alarms. On these occasions, it is usual that the operator leaves the alarm summary list and gets an analysis of the plant through the screens of the DCS (digital control system), seeking to understand the situation. The alarm summary list ceases to be a useful tool. In such cases, the operator might have the aid of a filter that would present the highest priority alarms and other information associated with them, enabling him to gain a better knowledge of the situation. This paper describes the interface of a system aimed to help the operator to have a more comprehensive knowledge of the process (a better situational awareness) during process upsets that cause alarm flooding, recovering the utility of the alarm layer to the safety of industrial processes.
\end{abstract}

Key words: Alarm summary list, alarm flood, situational awareness, ecological interface design.

\section{Introduction}

The human being is the supervisor of complex processes, located in industrial plants more and more automated, on which the operator's direct, continuous intervention is dwindling. The automatisms of those plants are designed to control process variations, considering normal operational situations and abnormal situations foreseen during the design. In spite of that, those automatisms, either in the safety layer or in the control layer, might not be sufficient in several moments, when human operator's intervention is expected to drive the process to its normal situation or prevent greater damages. That is a dilemma that could be named "automation paradox": on one hand, one of the reasons for process automation is to minimize human errors, taking people out of process control loops; but, on the other hand, as not all failures

Corresponding author: Eduardo Navarra Satuf, M.Sc., research fields: automation engineering and human computer interaction.E-mail: satuf@petrobras.com.br. are covered, we trust on operator's cognitive capacity to fill gaps of automation systems during abnormal situations and prevent greater incidents [1].

Alarm systems exist to help the operator to perceive an abnormal situation. Alarm systems are one of the process safety layers. When a fault happens, a fault foreseen in the process design, an alarm is activated requesting an operator's action. Often, several anomalies happen concurrently, related or not. Each anomaly can activate different alarms, in a cascade effect. Therefore, many alarms are simultaneously activated, competing for operator's attention. How can one operate at several fronts at the same time? One must choose which alarms to answer, implying to choose which not to answer. A current solution is to prioritize each alarm in design time. One possible step further might be the dynamic alarm prioritization, in real-time, based on a knowledge base previously established.

In this work, the motivating ideas for the interface of SAAI (intelligent alarms advanced system) and its 
operating interface are presented. The paper is organized as follows: Section 2 gives the reasons for the development of the software; Section 3 presents the important concept of situational awareness and its application; Section 4 presents the ecological interface design approach on some basic of ecological interfaces characteristics; Section 5 describes the current state of SAAI and the application of ideas from the EID (ecological interface design) framework; and, finally, Section 6 brings some concluding remarks.

\section{Motivation}

Oil production platforms and refineries are examples of complex systems (systems that comprise a large number of components interacting one with another, and where unexpected behaviour may emerge). Either production platforms, or refineries, both highly automated, have control rooms, locally or remotely, where the supervising consoles are located. Operators use those consoles to supervise the process plant and the control system performance, monitoring process variables and intervening when necessary.

A large amount of information (thousands of input or output points), and a large amount of automatisms and a variety of actuations over the plant are available at the control and automation system. In the last 20 or 30 years, the problem of information absence has diminished a lot. Information is available in the system, even though sometimes it is not displayed by the supervisor program ("the supervisory") - the operating interfaces.

A poll was made among experienced operators on refresher training at a process plant simulator of an oil production platform (Table 1). The simulator is part of the AmbTrei (process operators training environment) [2]. Eleven operators, with an average of eight years of work experience, answered if they agreed or not with statements related to the operating interface and to the alarm interface. They were asked about two scenarios: either with or without an alarm flood. A six-point scale was used (“ 1 ” for "do not agree” and "6" for "agree”). An alarm flood is the activation of alarms at a rate higher than it is possible for an operator to attend effectively [3].

Table 1 shows a great difference between the evaluations of the automation system in each of this two operating scenarios: "without alarm flood” and “with alarm flood”. Process alarm perception drops from $64 \%$ operating without an alarm flood to $9 \%$ during an alarm flood, undermining both the comprehension of current process state and the operator's projection capacity of the future state of the process. That means that $64 \%$ of the operators agreed (they marked " 5 " or " 6 " in the poll) that the operating interface they used helped the perception of an alarm and to what it refers when not in an alarm flood situation. Only 9\% continued to agree when asked about the perception of an alarm when in an alarm flood.

Although $82 \%$ of the operators did not agree that the interface had an information overload, only $9 \%$ says that the interface allows good perception during alarm floods. In other words, those $82 \%$ were considering the scenario without alarm flood, since, to $91 \%$, an alarm flood prevents them of perceiving alarms and an alarm flood is an overload of information by its own definition.

That excess of information during an alarm flood increases the cognitive load over the operator, since he will have to search for the piece of information of interest among several pieces of information without any interest on that moment. On those occasions, it is common that the operator leaves the alarm interface

Table 1 Results for a poll among oil production platform operators.

\begin{tabular}{|c|c|c|c|c|c|c|c|c|}
\hline Responses & $\begin{array}{l}\text { Perception of } \\
\text { alarms }\end{array}$ & $\begin{array}{l}\text { Perception in } \\
\text { alarm flood }\end{array}$ & $\begin{array}{l}\text { Compre- } \\
\text { hension }\end{array}$ & $\begin{array}{l}\text { Comprehension } \\
\text { in alarm flood }\end{array}$ & Projection & $\begin{array}{l}\text { Projection in } \\
\text { alarm flood }\end{array}$ & $\begin{array}{l}\text { Lack of } \\
\text { information }\end{array}$ & $\begin{array}{l}\text { Excess of } \\
\text { information }\end{array}$ \\
\hline "1" to "4" & $36 \%$ & $91 \%$ & $18 \%$ & $64 \%$ & $27 \%$ & $55 \%$ & $55 \%$ & $82 \%$ \\
\hline “ 5 ” or " 6 " & $64 \%$ & $9 \%$ & $82 \%$ & $36 \%$ & $73 \%$ & $45 \%$ & $45 \%$ & $18 \%$ \\
\hline
\end{tabular}


and looks for the cause of the anomaly on the process screens in the operating interface. That means that the alarm interface is not being useful.

Another issue is that the human capacity of information processing (i.e., the number of operators, their training and their experience) has not increased in the same proportion as the quantity of information offered by supervision systems. On the contrary, there is a trend for reducing the number of operators for each unit, as a result of automation mechanisms, and for safety reasons (decreasing hours of exposure to risk).

More (and more sophisticated) automatic systems require better operator's situational awareness [4], and the development of new operating interfaces becomes fundamental which shall promote that situational awareness and help operator's decision making (specially in the case of anomalies that can result in alarm flood).

This paper presents SAAI, which has the objective of giving better assistance using EID concepts.

\section{Situational Awareness}

SAAI's intention is to allow a better SA (situational awareness) to the operator attending to the alarms, i.e., the operator's understanding in order to make a better decision of what to do to drive the process back to a normal situation when an anomaly occurs.

The SA model presented in Fig. 1 was developed by Endsley [5] for aviation tasks, but it can also be applied to other domains that require people to monitor events, like process operation. This model comprises three levels: the perception of elements within an environment, the situation comprehension, and the projection of situation into the near future. There are several questionnaires to evaluate operator's SA [6].

As mentioned, during complex process operation, like oil production on an offshore platform, anomalies may occur that lead to alarm floods. On those occasions, keeping one's situational awareness may become particularly hard for the excess of alarms (information) calling operator's attention. Even when alarms are sorted by their priorities, those priorities were established in design time. So, those priorities may not reflect the current situation. Operators, when dealing with an alarm flood, leave the alarm list and go on analysing the process by the supervision screens (monitoring and actuating screens) trying to understand the situation [7]. The alarm list is not useful anymore.

In the poll, besides the alarm flood impairing alarm perception (64\% answered that operating interface was satisfactory; but that number drops to $9 \%$ in case of alarm flood), it also impairs comprehension and projection (from $82 \%$ to $36 \%$; and from $73 \%$ to $45 \%$ in case of alarm flood, respectively).

\section{EID Approach}

The EID approach was developed to support a rapid perception and correct interpretation of real-time information of complex socio-technical systems [8], as, for example, an industrial process supervised by an operator in a control room.

The EID can be summarized as follows [9]:

(1) control and observation screens developed to promote interaction through trend graphics (time-space signals);

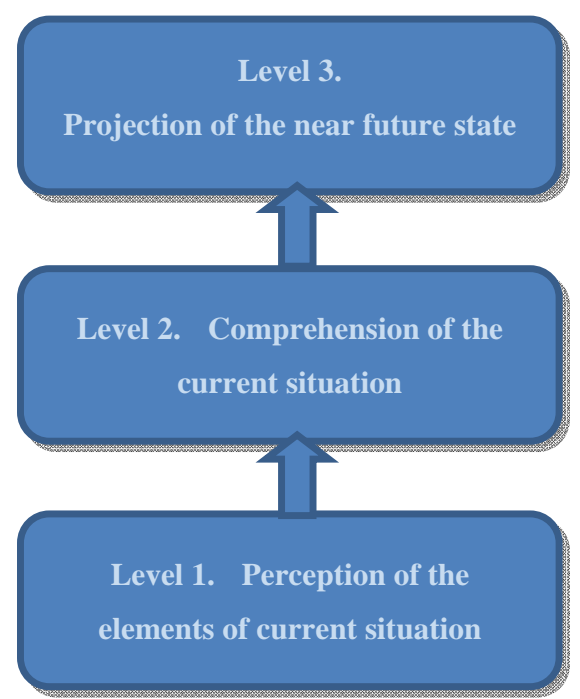

Fig. 1 Three levels model for SA. 
(2) consistent relationship between process properties and values provided by the interface;

(3) visualization of the process structure to function as a mental model that supports knowledge-based processing.

The "ecological" in "ecological interface" refers to the relationship between the operator and the environment (the process and the supervision system), opposing this framework to, e.g., a task-based interface framework.

The ecological interface should dynamically combine available information to help the rapid perception, comprehension and projection of the operational situation, and, therefore, facilitate the decision making process $[10,11]$. Research on operating interface for a nuclear power plant shows that ecological interfaces, in unanticipated situations, improves situational awareness compared to traditional interfaces or user-centred interfaces [12]. There is no reason to presume that it would be different in other situation under the same domain of "process control and supervision”. Coherently, as in Ref. [10], ecological interface can help to diagnose process malfunctions.

The ecological interface approach could be used for alarm systems interfaces to help operators to get an improved situational awareness and to allow, as much as possible, that knowledge-based tasks (that are more cognitively demanding) turns into skill-based tasks [8].

\section{Description of SAAI}

As mentioned, alarm means a process anomaly. An alarm flood means several process anomalies that can be correlated. An alarm flood, then, is a moment of stress to the process and to the operator (who needs to know the cause of the abnormal situation and decide what to do to drive the process to a normal situation or to mitigate the consequences of the malfunction).

Current automation systems at oil production platforms or oil refineries show alarms as a list and as icons on operating screens. Alarms in the list are shown in a reverse chronological order: the top of the list is the most recent alarm. Colour prioritization is also used. Those alarm lists, during an alarm flood, increase quickly, and operators do not have time to read and interpret all information. Thus, they leave that alarm list interface and navigate through operating screens, that also contain alarm animated icons, trying to diagnose the problem. Current interfaces, then, can delay the decision-making.

Another issue is shown by some incident analysis: the human being, after finding one possible cause for a process problem, tends to take action to correct the situation, but he will hardly change his mind, even if his actions are worsening the scenario [1]. If there is no intervention from a supervisor, less than $1 \%$ of the occurrences will result in rethinking actions to be taken. That is why it is so important that the interface minimize chances of a wrong initial diagnosis.

SAAI is proposed as an independent interface (from the plant operation systems), that will process the alarms and other variables (either analogical or digital variables), providing information to allow a quick and correct perception about the process status, and a correct decision making. The advantage of having an independent alarm system is its flexibility, for implementing algorithms like an expert system inference motor. The "expertise" of that system would come from several rules in its knowledge base, defined by operators and engineers of that site.

Another point is that the SAAI interface can be tailored and will be visible during all the diagnostic process. In a way, we are going back to a past time and implementing a modern and more resourceful version of an alarm annunciator. SAAI does not suppress the alarm interface of the existent supervision system, but it adds an external layer that dynamically processes active alarms, and presents them in order to help operators in decision-making.

The prioritization algorithm of SAAI is based on a "static priority" given to each alarm in design time. The static priority is given according to the probability 


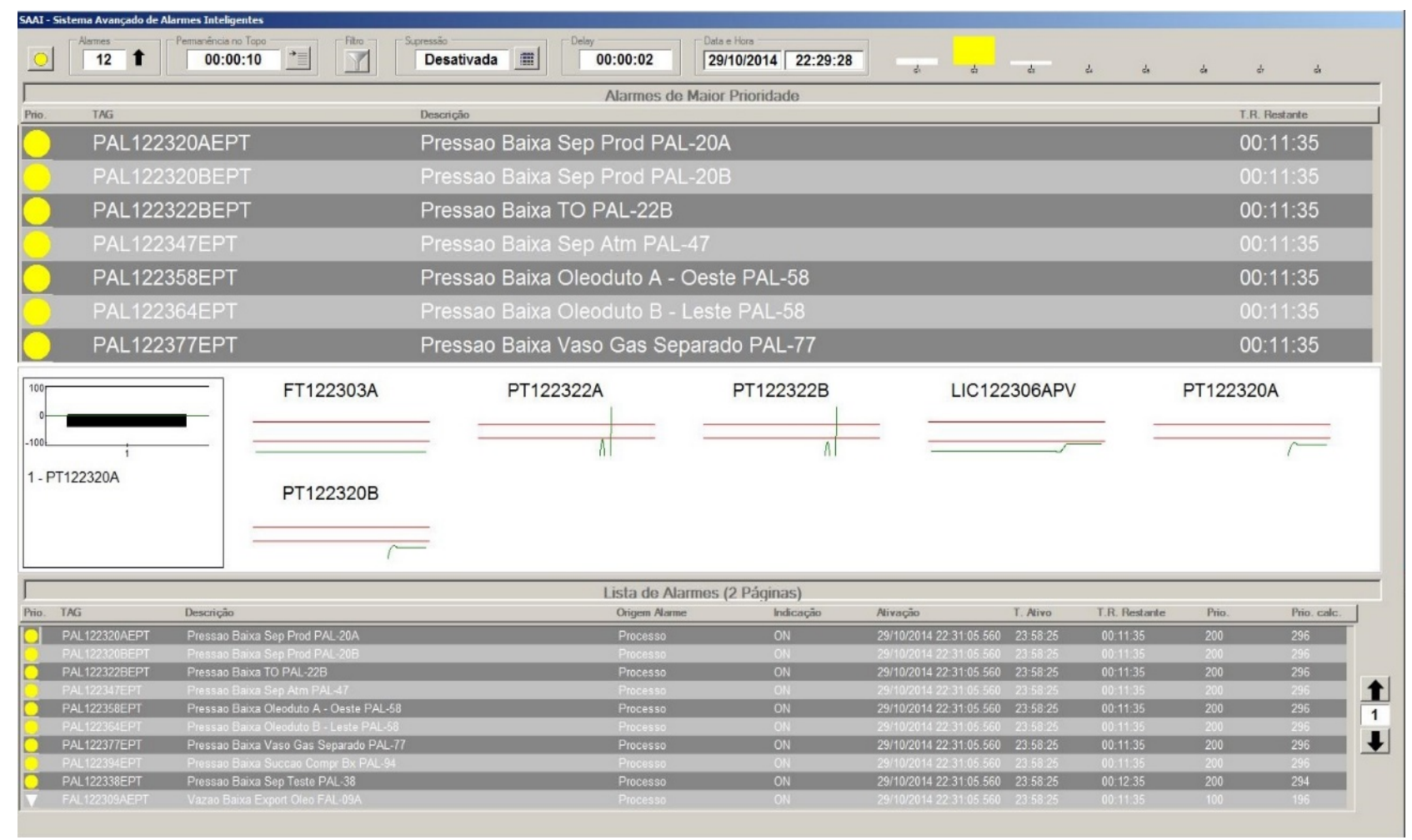

Fig. 2 Alarm interface of SAAI.

and the severity of the consequences (to people, to environment or to the plant) in case nothing is done when the respective alarm is activated [3].

Three levels of priority are commonly used: low, medium or high priority. There may be a fourth level, critical priority, with special features, comprising at most 20 to 40 alarms from a universe of over 2,000 configured alarms. A recommendation to current supervision systems would be to show the critical alarms on an alarm panel for that purpose.

SAAI algorithm recalculates dynamic priorities for each alarm according to the available time to the operator to attend to the alarm. SAAI might also take into account, in the alarm priority calculus, other process variables evaluated in real-time, like values of other analog variables or equipment condition (e.g., number of pumps that are either on or off). Correlated alarms could also be suppressed or filtered in SAAI, which could show a more significant alarm (at that particular moment) replacing those suppressed.

Fig. 2 illustrates the SAAI interface. This tool is said to be "advanced" because it is installed as a layer "over" the existent process supervision system, receiving signals from this one. The alarms are said to be "intelligent" because of SAAI algorithm of alarm dynamically prioritization and alarm generation according to its own KB (knowledge base).

On the upper half of Fig. 2, the seven most important alarms (at current time) are shown in a list, the most important one being at the top. A newly activated alarm which has the same static priority of the top one (e.g., they both are configured as "low" priority) may gain the top position or not, depending on its available time to be attended.

On the central band of the image, trend graphics and others show the behaviour of variables related to the top alarm in order to facilitate the perception of the relevant status of the process at the time and to increase operator's situation awareness, helping in the diagnosing process.

When an alarm in the list is selected, its related graphics are shown on another screen, without hiding 
the main one.

The lower half shows a complete list of all active alarms (included in the SAAI database). Other auxiliary informations are displayed, as the system time and the total number of active alarms.

SAAI seeks to synthesize the needed observation as graphics on a single screen, giving, as much as possible, a holistic vision to the operator and supporting his decision. The relevant process properties (variables) are made visible, in a way showing their relationship with the alarm, trying to make explicit a reference model that would help the operator to build his own mental model. These are relevant characteristics from an EID point of view.

In addition, SAAI allows a configuration in order to show the status of the relevant portion of the process (to the current top priority alarm), without restricting (nor inducing) the response procedure.

SAAI has been installed and has its functionalities tested on a dynamic simulator, part of an operator training system called AmbTrei [2]. The process plant of an oil production platform is modeled. SAAI is connected to the plant supervision system. Questionnaires to evaluate the gains to the situational awareness with an external interface are being submitted to the operators being trained.

\section{Conclusions}

A software for alarm prioritizing and filtering, SAAI, was described. The goal of the software is to increase operator's situational awareness, and, thus, help the decision-making. The software interface has some features of the EID framework.

As seen in a poll among operators from oil production platforms, current supervision systems, during alarm floods, do not provide a satisfying alarm interface and do not help to construct situation awareness on those occasions.

The proposal of this work is the utilization of an advanced alarm interface (SAAI) independent from supervision systems, which would process alarms, providing information for a rapid perception of the process status. SAAI includes a KB, which has rules for filtering, prioritization, suppressing and generating alarms. An investigation to evaluate operators' situational awareness using advanced interface like this is under way.

\section{Acknowledgments}

The authors would like to thank the colleagues at Petrobras who contributed to the development and testing of SAAI; to the trainees and instructors at AmbTrei, for their time; to the support team of SENAI-Benfica (Rio de Janeiro); and to the SAAI development team at COPPE. The first author would also like to thank the professor Eugenius Kaszkurewicz (UFRJ).

\section{References}

[1] Reason, J. 1990. Human Error. UK: Cambridge University Press.

[2] Pereira, A. C. 2009. “Operator Trainer System for the Petrobras P-26 Semi-submersible Oil and Gas Production Unit.” In Proceedings of the 10th International Symposium on Process Systems Engineering, 1959-64.

[3] EEMUA (Engineering Equipment and Materials Users Association). 2007. Alarm Systems-A Guide to Design, Management and Procurement.

[4] Schmitt, K. 2012. "Automations Influence on Nuclear Power Plants: A Look at Three Accidents and How Automation Played a Role." Work: A Journal of Prevention, Assessment and Rehabilitation 41: 4545-51.

[5] Stanton, N. A., Chambers, P. R. G., and Piggott, J. 2001. "Situational Awareness and Safety." Safety Science 39 (3): 189-204.

[6] Salmon, P. 2006. "Situation Awareness Measurement: A Review of Applicability for C4i Environments.” Applied Ergonomics 37 (2): 225-38.

[7] Li, X. 2011. "The Control Room Operator: The Forgotten Element in Mineral Process Control.” Miner. Eng. doi:10.1016/j.mineng.2011.04.001. (in press)

[8] Skraaning, G. 2005. "The Ecological Interface Design Experiment.” Cognitive Engineering Laboratory, University of Toronto. Accessed December 3, 2013. http://gypsy.mie.utoronto.ca/publications/technical-report s.htm.

[9] Rasmussen, J., and Vicente, K. J. 1989. “Coping with Human Errors through System Design: Implications for Ecological Interface Design.” International Journal of 
Man-Machine Studies 31 (5): 517-34.

[10] Jamieson, G. A. 2007. "Ecological Interface Design for Petrochemical Process Control: An Empirical Assessment. Systems, Man and Cybernetics.” IEEE Transactions on Systems and Humans Part A 37 (6): 906-20.

[11] Jamieson, G. A., and Vicente, K. J. 2001. "Ecological Interface Design for Petrochemical Applications: Supporting Operator Adaptation, Continuous Learning, and Distributed, Collaborative Work.” Computers \& Chemical Engineering 25 (7): 1055-74.

[12] Burns, C. M., Skraaning, G., Jamieson, G. A., Lau, N., Kwok, J., Welch, R., and Andresen, G. 2008. "Evaluation of Ecological Interface Design for Nuclear Process Control: Situation Awareness Effects.” Human Factors: The Journal of the Human Factors and Ergonomics Society 50 (4): 663-79. 